\title{
Infrastructure numérique et environnement :
}

\section{L'impossible domestication de l'effet rebond}

\author{
Fabrice Flipo \\ Maître de Conférences \\ Institut TELECOM, TELECOM \& Management Sud Paris \\ ETOS / CEMANTIC \\ 91011 Evry (fabrice.flipo@it-sudparis.eu) \\ Cédric Gossart \\ Maître de Conférences \\ Institut TELECOM, TELECOM \& Management Sud Paris \\ ETOS / CEMANTIC \\ 91011 Evry (cedric.gossart@it-sudparis.eu)
}

\section{Introduction}

Les technologies numériques de l'information et de la communication (TNIC) se généralisent dans un grand nombre de domaines de la vie collective et domestique. Les puces envahissent notre quotidien : automobiles, PDA, lecteurs MP3, etc. Les TNIC véhiculent une image de légèreté et d'absence de friction. Les coûts de transaction et les frais de transport sont ramenés quasiment à zéro. Leur effet sur l'écologie planétaire semble être nul. Mieux, elles permettent d'observer la planète et ses évolutions de loin, sans avoir l'air d'y toucher. Elles permettent la démocratie, la croissance, voire l'inclusion sociale. Les TNIC seraient-elles " par nature » favorable à l'écologie et au développement durable ? Cet article entend au contraire montrer que non seulement tel n'est pas le cas, les TIC ayant une existence matérielle de plus en plus encombrante, mais en plus la régulation de cette empreinte écologique ne semble pas aller dans le bon sens, les efforts dans le domaine de l'éco-efficacité étant appelés, de manière structurelle, à être contrebalancés par "l'effet rebond»- du moins tant que les conditions inhérentes à la compétition économique restent inchangées. L'analyse de l'effet rebond, ou plutôt des effets rebond, révèle en effet des régulations collectives qui, si elles ne sont pas modifiées, annihileront toujours les efforts locaux faits dans le domaine de l'éco-efficacité.

\section{Qu'est-ce que l'effet rebond?}

L'effet rebond correspond au fait que l'accroissement des consommations de matières et d'énergie induit par l'utilisation généralisée des TIC efface largement les réductions de l'empreinte écologique obtenues par unité de produit. De nombreuses études ont étudié l'effet rebond (ER) ${ }^{1}$; elles s'accordent sur une typologie comportant trois ou quatre catégories. Greening et al (2000) proposent une typologie appliquée aux services énergétiques qui prend en compte les dimensions micro et macroéconomiques de ce phénomène. Le premier type d'ER, l'ER direct, joue au niveau microéconomique d'un service énergétique. Lorsque l'on réduit l'intensité en énergie d'un service énergétique, son prix tend à diminuer et son coût pour le consommateur final à baisser. Dès lors, soit ce dernier utilise l'économie résultante pour consommer plus du service concerné (effet substitution), soit il estime avoir atteint un niveau de consommation de ce service satisfaisant, auquel cas et il affecte cette économie à d'autres fins (effet revenu). Suite à ce dernier effet, le choix d'une consommation accrue d'autres services ou biens génère un deuxième type d'ER, qui correspond à une consommation induite de matière ou d'énergie. L'individu pourra par exemple décider de voyager plus loin, une entreprise pourra en profiter pour étendre ses activités de

\footnotetext{
${ }^{1}$ Pour un résumé voir Herring $(1998,2004)$ paru dans un numéro spécial de la revue « Energy Policy », ainsi que Schipper \& Grubb (2000), Vikström (2004), Grepperud \& Rasmussen (2004), IEA (2005), Sorrell \& Dimitropoulos (2005).
} 
production, qui sont rarement énergétiquement neutres. Un troisième type d'ER reflète l'impact des ajustements de prix et de quantités consécutifs aux deux ER précédents sur les agrégats de consommation et d'investissement des consommateurs privés et publics. Bien moins avéré que les précédents, il opère notamment à travers les ajustements de prix sur les marchés d'approvisionnement en matière et en énergie. Il peut contribuer à renchérir les biens et services intermédiaires et favoriser l'essor de la croissance économique en général et des secteurs gourmands en énergie en particulier. Enfin, le quatrième type d'ER regroupe des effets dits de transformation, qui ont le pouvoir de changer les préférences des consommateurs, d'altérer les institutions sociales, et de modifier l'organisation de la production. Les auteurs ne citent pas d'étude de cas pour le secteur des TNIC. Quel serait l'effet d'une baisse de l'intensité énergétique des serveurs informatiques sur les quantités vendues? Tout comme dans le cas des avions soidisant économes en énergie ${ }^{2}$, on peut craindre un fort ER du premier type, confirmé par le succès du Boeing 787 qui se vend comme des petits pains alors que le baril de pétrole se rapproche des 100 dollars $^{3} \ldots$ Peu d'études portent sur l'ER et les TNIC, mais on imagine facilement l'argumentaire vert des fabricants de serveurs informatiques très peu gourmands en énergie ${ }^{4}$. Dès lors le cycle de vie de l'appareil est réduit, de "bien durable " il devient peu à peu un " consommable » que l'on considère normal de renouveler alors qu'un des moyens principaux d'améliorer l'éco-efficacité des services rendus par les appareils est au contraire d'accroître la durée de vie ${ }^{5}$.

Les études sectorielles de l'ER portent souvent sur le secteur énergétique et des transports. Haas et Biermayr (2000) comparent différentes approches utilisées pour évaluer l'ampleur de l'ER au niveau du chauffage en Autriche. Ils mettent en avant un ER non négligeable de 20 à $30 \%$. Herring et Roy (2002) avancent que l'éducation à distance génère $90 \%$ de consommation d'énergie et d'émissions de $\mathrm{CO}_{2}$ en moins que des cours dispensés sur un campus, mais soulignent en revanche qu'à cause de l'ER ce n'est pas le cas de l'éducation à distance en comparaison avec celle basée sur le papier. Concernant le rôle des véhicules hybrides dans la réduction de la consommation énergétique, Haan et al. (2007) avancent que les réductions d'impôts liées à l'achat de ces véhicules conduisaient à une augmentation significative de leurs ventes. Estimant l'ER pour les véhicules à moteur, Small et Van Dender (2007) concluent à des ER sensiblement différents selon le niveau du prix de l'énergie et qui varient entre 2,2 et $22,2 \%$. Sur le long terme, l'ER est significatif et tend à remettre en cause le succès des politiques d'efficacité énergétiques des véhicules. En effet, ils ont calculé que pour les Etats-Unis et au cours du dernier tiers du $20^{\mathrm{e}}$ siècle, ces politiques ont accru de 20 à $25 \%$ le nombre de kilomètres parcourus en véhicule, ce pourcentage étant très sensible au revenu.

On trouve aussi des études macroéconomiques dont nous ne parlerons pas ici ${ }^{6}$.

\section{Le poids écologique croissant des TNIC}

Loin de la représentation immatérielle des TNIC et de leur apparente légèreté, ces dernières nécessitent beaucoup de ressources pour leur fabrication. Produire un PC de $24 \mathrm{~kg}$ nécessite $240 \mathrm{~kg}$ de carburants fossiles, $22 \mathrm{~kg}$ de produits chimiques et 1500 litres d'eau, soit en proportion plus que pour fabriquer une voiture (Kuehr et Williams, 2003). Dans le cas d'un téléphone portable 3G, Faist Emmenegger et al. (2003) montrent que l'impact environnemental est réalisé à $60 \%$ par la fabrication du téléphone, tandis que la phase d'usage ne constitue que $30 \%$ du total. Hilty (2008) souligne que l'impact des TNIC sur l'environnement dépend largement du contexte dans lequel elles sont utilisées, ce qui inclut le comportement des utilisateurs (on peut par exemple

\footnotetext{
${ }^{2}$ Source : http://news.bbc.co.uk/2/hi/business/6285350.stm.

${ }^{3}$ Source : http://www.usatoday.com/travel/flights/2006-06-08-boeing-787_x.htm.

${ }^{4}$ Voir par exemple http://news.mongabay.com/2006/1204-dell.html ou http://www.pcmag.com/article2/0,2817,2271432,00.asp.

${ }^{5}$ Voir ACV téléphone portable : http://www.ademe.fr/internet/telephone-portable/Site-web/index.html.

${ }^{6}$ Voir notamment Berkhout \& al. (2000), Barbera \& al. (2007), Binswanger (2001), Birol \& Keppler (2000), Mizobuchi (2008).
} 
laisser son PC branché 24 heures sur 24, ou le mettre en veille dès qu'on ne s'en sert pas pendant plus de 5 minutes).

Entre 1993 et 2000, le nombre de PC par habitant terrestre a augmenté de 181\%. En avril 2002, le milliardième PC a été livré (Kuehr et Williams, 2003), et l'on comptait 4 milliards d'abonnements de téléphones portables en $2008^{7}$. Comme toutes les infrastructures, les autoroutes de l'information demandent à être entretenues et donc régulièrement alimentées en matériaux et en énergie. Cremer et Eichhammer (2003) estiment que leur consommation d'énergie représente entre 3 et $10 \%$ de la consommation électrique allemande. De même, Aebischer et Roturier (2006) soulignent que la consommation des télécommunications mobiles est générée à $90 \%$ par l'infrastructure et $10 \%$ par le terminal. Quelle que soit la demande, les serveurs tournent 24 heures par jour dans des salles climatisées. En France, sur les $75 \%$ d'augmentation de la consommation électrique dans les ménages depuis 1990, une part importante est liée à l'expansion des TNIC $^{8}$.

Mis sur le marché, les produits finissent tôt ou tard par en sortir pour devenir des Déchets d'Equipements Electriques et Electroniques (DEEE) ${ }^{9}$. En 2007, le volume d'équipements TNIC mis sur le marché était de l'ordre de 100000 à 2000000 tonnes dans les principaux pays européens. Chaque année, entre 20 et 50 millions de tonnes de DEEE sont produits dans le monde ; chaque citoyen de l'Union Européenne s'est débarrassé de $25 \mathrm{~kg}$ de ces déchets. La croissance en volume est de 3 à $5 \%$ par an, un taux quasiment triple du taux des déchets classiques, notamment du fait de l'obsolescence accélérée des produits. D'après l'US EPA (2001), la durée de vie des ordinateurs a chuté, passant de 6 ans en 1997 à deux ans en 2005. Le PNUE (2005) estime que les téléphones portables ont une durée de vie de moins de deux ans dans les pays industrialisés. Plus de $80 \%$ du flux de DEEE des USA est exporté vers des pays d'Asie mal équipés en capacités de traitement comme la Chine ou l'Inde (Toxic Link, 2003). Les conditions de recyclage peuvent être dramatiques: un échantillon d'eau de la rivière Lianjiang, proche d'un village de recyclage chinois a révélé des taux de plomb entre 190 et 2400 fois plus élevés que les standards préconisés par l'Organisation Mondiale de la Santé et 16 fois plus élevés pour l'antimoine (BAN et SVTC, 2002). II a été observé une concentration de dérivés chlorés deux fois plus élevée que la normale dans le lait des mères vivant près d'un site de recyclage de DEEE ${ }^{10}$, des déchets dangereux tombant sous le coup de la convention de Bâle. La Commission de Coopération Environnementale de l'Amérique du Nord, qui dépend de I'ALENA (Accord de Libre-Echange Nord-Américain), évoque même une « menace mondiale » (Ostroff, 2007).

\section{Effet rebond et TNIC}

Encore peu étudiés dans la littérature, les ER que peuvent générer les TNIC sont pourtant nombreux, et cela d'autant plus que les politiques publiques font de plus en plus appel aux TNIC pour réduire notre consommation d'énergie et de ressources naturelles. Par exemple, EDF est en train de remplacer les 35 millions de compteurs électriques par des compteurs dits " intelligents » qui permettront aux ménages de suivre leur consommation en temps réel ${ }^{11}$, et éventuellement d'ajuster leur consommation à la baisse et d'économiser de l'argent. Equation "gagnantgagnant » permettant de hisser les TNIC et les politiques publiques les soutenant au sommet du palmarès des technologies "vertes "? Tout dépend de la manière avec laquelle on évalue ces technologies et politiques. En effet, le ménage dont la facture a été réduite peut alors choisir de plus chauffer son appartement ou de changer son écran de télévision cathodique par un écran plat très gourmant en énergie et qui se recycle très mal ; c'est là qu'apparaît l'effet rebond. Herring et Roy (2007) soulignent que plus les consommateurs sont sensibles aux variations du prix de l'énergie (forte élasticité), plus la baisse, par exemple, de ce prix, se traduira par une augmentation de leur consommation énergétique (effet de substitution). Dans ce contexte, tout l'enjeu des politiques de réduction de la consommation énergétique sera par exemple de faire en sorte que les

\footnotetext{
${ }^{7}$ Source : http://www.itu.int/newsroom/press releases/2008/29.html.

${ }^{8}$ Source : http://www.industrie.gouv.fr/energie/statisti/rt energies usage.htm.

${ }^{9}$ Pour en savoir plus sur les DEEE, voir http://www2.ademe.fr/servlet/KBaseShow?catid=14687.

${ }^{10}$ Source : http://www.iges.or.jp/en/ltp/pdf/activity08/20 wong.pdf.

${ }^{11}$ Source : http://www.01net.com/editorial/370728/les-compteurs-electriques-communicants-d-edf-mis-sur-les-rails.
} 
économies réalisées grâce au progrès technologique ne se traduiront pas par des choix de dépense consommant beaucoup d'énergie...

Un autre type d'ER lié aux TNIC suggère que l'amélioration des performances qu'elles génèrent tend à accroître la consommation du bien ou service dont la productivité a été augmentée (Plepys, 2002). Par exemple, un moteur à injection électronique permet de maîtriser la consommation d'essence et constitue donc une source d'avantage comparatif... qui va booster les ventes du véhicule en question. Plepys (2002) donne de multiples exemples de tels ER qui soulignent par exemple combien Internet peut être un puissant vecteur d'accroissement de la consommation en facilitant l'identification, la comparaison, et l'achat de biens et services.

Pour Herring et Roy (2007) la certitude d'un ER suggère que les politiques de soutien à l'innovation visant à réduire la consommation énergétique et les émissions de GES sont vouées à l'échec. II suggère d'utiliser d'autres instruments comme la taxation ou la réglementation en vue " d'internaliser les externalités " et faire en sorte que les comportements vertueux soient non seulement possibles mais même récompensés - tel est, dans un autre secteur, l'exemple du " bonus-malus », dont le signal est clair et a été extrêmement efficace. Encore faut-il utiliser ces instruments à bon escient car ils ne sont jamais neutres sur le plan de la justice sociale, comme en témoigne à nouveau l'exemple du « bonus-malus » automobile.

En définitive, le succès des politiques utilisant les TNIC pour, par exemple, réduire notre consommation énergétique reste très largement tributaires des préférences de l'acteur individuel. Cela souligne l'importance de politiques permettant au consommateur, final ou intermédiaire, dont le souci écologique est avéré12, de pouvoir exprimer ses préférences de manière cohérente. L'un des freins évidents à un tel comportement est qu'un choix plus écologiste peut se traduire par une désaffection de certains marchés au profit d'autres marchés, ce qui n'est pas dans l'intérêt des acteurs du marché victime de la chute d'activité qui va en résulter. Par exemple les consommateurs de portables pourraient se retourner vers plus de papier (renouvelable) ou de téléphonie fixe, dont l'obsolescence plus lente est cohérente avec une volonté de durabilité. Se pose alors la question des limites aux besoins matériels dans les pays riches, ce qui soulève des interrogations profondes sur la régulation de nos économies ${ }^{13}$. Examinons maintenant comment I'ER peut jouer à chacune des phases du cycle de vie des TNIC.

\section{ER et cycle de vie des produits}

L'évaluation de la contribution des TNIC à l'environnement porte sur l'ensemble du cycle de vie de ces technologies, depuis leur conception jusqu'à leur fin de vie en passant par leur utilisation. A chacune de ces phases, les causes de l'effet rebond comme révélateur des impasses du débat actuel où les TNIC éclipsent le changement de style de vie dans la recherche d'un développement durable. La fin de vie ayant déjà été abordée dans la section 3, nous insisterons ici sur la conception et l'utilisation des TNIC.

\subsection{Conception des TNIC et ER}

A moins d'en être obligés par la législation comme dans le cas des directives européennes RoHS ou EuP ${ }^{14}$, les producteurs de TNIC anticipent rarement les impacts environnementaux pourtant connus de ces technologies. Certains d'entre eux ont fait des efforts de prévention avec l'écoconception, qui permet de limiter les impacts environnementaux en amont, mais ces efforts sont déjà assez anciens pour certains d'entre eux et pas forcément guidés par le souci écologique. Le problème de surchauffe et la part grandissante de la facture énergétique dans les frais d'exploitation d'un data centre ont motivé une partie d'entre eux à trouver des moyens de réduire la consommation d'énergie de leur produits. Mais dans le même temps, d'autres choix

\footnotetext{
${ }^{12}$ Voir le sondage ADEME : http://www2.ademe.fr/servlet/getDoc?cid=96\&m=3\&id=52401\&ref=21479\&p1=B.

${ }^{13}$ Sur la diffusion du mouvement de la simplicité volontaire, voir le numéro $n^{\circ} 896$ du 3 janvier 2008 de Courrier International intitulé « Travailler moins pour gagner moins et vivre mieux », ainsi que la conférence http://events.it-sudparis.eu/degrowthconference.

${ }^{14}$ A propos de ces directives, voir http://www.codde.fr/fr/Reglementation.html.
} 
contradictoires également motivés par la réduction des coûts sont faits, comme celui de l'accélération de l'obsolescence des produits ou les sommes considérables investies dans le marketing et la publicité. Les publicités qui mettent en avant un produit " plus vert » que ses concurrents tendent à déculpabiliser les acheteurs et à le rendre relativement plus attractif. Les ventes de ce produit se multiplient, et l'impact environnemental global de l'ensemble des nouvelles ventes efface de loin la réduction marginale de la pollution par unité produite : voilà l'une des sources banales de l'effet rebond, que l'écoconception ne permet pas de résoudre.

L'écoconception pose elle-même de sérieux problèmes. Les analyses de cycle de vie sur lesquelles elle se base n'offrent pas de solution simple. Les produits TNIC sont les mêmes dans le monde entier, mais les capacités de traitement varient énormément selon les territoires. Pour des pays qui ne savent gérer que les déchets inertes et les déchets organiques, les PC devraient être réalisés uniquement dans ces deux types de matériaux. Or rien n'indique que cela est possible. Les enquêtes de terrain montrent que les téléphones portables réutilisés perdent de la valeur et finissent dans les mains des plus pauvres qui sont situés... dans les campagnes, à l'endroit le plus éloigné des capacités de traitement ou de réparation (Flipo et al., 2006). L'écoconception d'un produit a de fortes implications au niveau d'un territoire, mais sa conception est totalement déterritorialisée, mondialisée. Le grand écart entre les deux niveaux sera difficile à combler sans toucher aux performances des appareils - ce qui aura des implications importantes en termes de service rendu. N'est-ce pas à nouveau l'une des raisons de l'effet rebond - qu'un produit ignore la diversité des situations à l'échelle mondiale ? Qu'un produit soit lancé par les pays à fort contenu technologique, sans tenir compte des conséquences dans les pays à bas contenu technologique ? C'est l'ensemble du système de gouvernance des déchets qui pose problème. Les filières de collecte sélective et les déchetteries n'ont pas inversé la tendance à l'augmentation des déchets produits, contrairement à ce qui était promis. En effet, d'après les données de l'INSEE si le volume de déchets ménagers a augmenté de $75 \%$ entre 1980 et 2000, le revenu des ménages n'a lui augmenté que de $45 \%$ et la population que de $10 \%$. L'écoconception est restée marginale et n'a réglé ni le problème de la collecte ni celui de l'élimination finale.

Quant à la miniaturisation, elle n'entraîne guère de réduction de l'impact écologique des produits. II faut de très grosses machines pour aller dans l'infiniment petit - que l'on pense aux gigantesques synchrotrons qui ont été construits pour descendre au niveau de la mécanique quantique. En réalité cet impact devient invisible : pendant que le poids est réduit, c'est l'empreinte écologique " amont » et « aval » des produits qui s'accroît. La solution ne semble " gagnante-gagnante » que parce qu'il existe un tiers exclu masqué. Certains services au sein des constructeurs en sont un peu conscients et certains d'entre eux tentent de produire des matériels selon les règles de l'écoconception. C'est notamment le cas de Nokia, mais comme le montre le classement réalisé par Greenpeace les performances environnementales des constructeurs sont très variables et changent dune année sur l'autre ${ }^{15}$. Mais le pouvoir des constructeurs en la matière est limité. Et les clients ne cherchent pas vraiment à utiliser des produits "verts ", ils considèrent en général ces labels comme étant des arguments commerciaux qui ne sont pas dignes de confiance (Attané, 2002).

\subsection{L'utilisation des TNIC : la redoutable question de la substitution}

La manière avec laquelle les TNIC sont utilisées peut réduire leurs impacts environnementaux, par exemple en utilisant le mode veille de son ordinateur ${ }^{16}$. Mais les promoteurs de ces technologies mettent surtout en avant leur capacité à " dématérialiser » l'économie, dont la croissance ne repose alors plus sur des atomes mais sur des octets perçus comme immatériels. Berkhout et Hertin (2001) ont proposé de hiérarchiser les effets des TNIC en trois catégories (respectivement : effets positifs ; effets négatifs) :

- Effets de premier ordre : applications environnementales des TNIC (monitoring); impacts écologiques des TNIC (DEEE).

\footnotetext{
${ }^{15}$ Source : http://www.greenpeace.org/international/campaigns/toxics/electronics/how-the-companies-line-up.

${ }^{16}$ La consommation résiduelle est alors plus faible mais non négligeable : voir http://www.01net.com/editorial/385921/les-pc-devront-consommer-moins-en-mode-veille.
} 
- Effets de second ordre: dématérialisation (administration électronique); changements structurels (augmentation de la quantité globale de produits facilitée par les TNIC).

- Effets de troisième ordre (substitution) : changements dans les modes de vie (consumérisme « vert »); effet rebond (augmentation de la température du logement).

Les discours politiques s'intéressent surtout aux effets positifs de premier et de deuxième ordre. Les TNIC permettraient par exemple de substituer "les bits aux atomes " (réduction des transports). Gendron (2002) a montré qu'identifier développement durable et entrée dans une ère high-tech à empreinte écologique légère est un trait commun de l'imaginaire des élites canadiennes. Le mythe de la dématérialisation ne répond pas à la question, il se défausse à nouveau via un tiers exclu: les pays en développement, qui subissent l'essentiel de l'impact écologique lié à l'activité des industries qui produisent les biens utilisés pour entretenir notre mode de vie (Zuindeau, 2005).

Certains pensent que les TNIC vont favoriser une réduction de la consommation par accroissement de l'efficacité (Laitner, 2005) tandis que d'autres pensent que la consommation va être accrue de plusieurs points de pourcentage (Cozzi, 2002). Au centre des débats, la question de la substitution pose en réalité deux questions autrement plus redoutables que les solutions techniques proposées par la théorie commode de la dématérialisation.

La première est la question de la substitution matérielle : on peut dans certains cas remplacer une matière par une autre, mais il y aura des limites écologiques, sociales et économiques. Ecologiques, car la substitution remplace une pollution par une autre ; sociales, car une ressource remplace une autre mais souvent à un coût supérieur, ce qui, dans un contexte de stagnation du pouvoir d'achat, ne manque pas de poser des problèmes ; économiques car la fluctuation des prix des matières premières déstabilise le cadre dans lequel les entreprises opèrent. Daly et Cobb (1989) ont montré il y a longtemps déjà que la question d'une économie écologique n'était pas un problème de substitution mais un problème d'échelle. L'enjeu des écosystèmes pose des questions normatives graves car les dégâts peuvent être fort avancés voire même irréversibles avant que les sociétés ne réagissent, comme l'a montré Diamond (2005). Entre l'effondrement des effectifs d'une population et le moment où l'on peut se rendre compte du danger a lieu une phase trouble et cruciale d'élaboration sociale des alternatives possibles, qui prend par exemple aujourd'hui la forme du débat sur la décroissance.

Ce qui nous mène à la seconde question, qui n'est évidemment pas indépendante de la première : la substitution sociale et sociétale. Prenons quelques exemples pour illustrer le propos. Le télétravail peut remplacer le travail nous dit-on, l'un peut se substituer à l'autre avec une réduction de l'impact écologique. Ce dernier résultat resterait à démontrer ${ }^{17}$. En effet, l'enjeu social apparaît de manière évidente dès lors que l'on s'intéresse aux effets réels, et non supposés, du télétravail sur le travail : les télétravailleurs sont bien plus sujets au surtravail que les autres. La corrélation est nette puisque plus de $80 \%$ des travailleurs voient leur temps de travail augmenter, alors que moins de $10 \%$ le voient diminuer (ECATT, 2000). Et ce n'est là que l'un des aspects du problème, l'éloignement des lieux collectifs ayant bien d'autres conséquences. Autre exemple: quand le Wuppertal Institut (2003) montre que le téléchargement est moins " impactant » que l'achat de $C D$, a-t-on là une solution qu'il suffirait de généraliser ? Rien n'est moins sûr : le bilan écologique flatteur n'est au rendez-vous que si l'usager n'utilise pas son graveur pour obtenir son CD. Le choix qui s'offre à nous doit donc être reformulé : il n'y a pas lieu de choisir entre la solution " propre » et la solution "sale ", comme si les deux étaient équivalentes, mais de choisir entre " le téléchargement à volonté avec interdiction de graver les CD » ou " l'achat rationné de CD en magasin » - la seconde solution offrant la possibilité d'être décentralisée et fortement modulable en fonction des conditions socio-écologiques.

Un tel choix est lourd de conséquences et pourtant ni le consommateur ni le citoyen ne rencontre nulle part de lieu où se confronter à ces alternatives. Quand il achète un produit, il contribue à

\footnotetext{
${ }^{17}$ Le mouvement de « dématérialisation » s'accompagne aussi d'une délocalisation des imprimantes, etc. qui au final consomment peut-être plus que les anciens systèmes, de même que le téléphone fixe consomme moins que le GSM qui consomme moins que la $3 G$.
} 
construire une infrastructure dont il sera ensuite dépendant, mais sur le moment c'est bien un produit et non une infrastructure qu'il acquiert. Quand il vote, personne ne lui parle des conséquences écologiques à long terme des politiques numériques, au contraire c'est un concert de louanges aux conséquences bénéfiques du " progrès technique », qu'on ne saurait décemment critiquer. L'usage d'outils comme les ACV opacifie le débat.

\section{Effet rebond et politiques publiques}

Nous avons montré dans les sections précédentes que l'ER était un obstacle difficilement surmontable pour que les TNIC contribuent au développement durable. Cela peut s'expliquer par au moins deux raisons. La première est que l'ER n'est pas intégré dans l'évaluation des politiques publiques visant à utiliser les TNIC pour mettre en œuvre un développement durable. Si l'on examine par exemple la communication de la Commission Européenne ${ }^{18}$ prônant la diffusion des TNIC pour améliorer l'efficacité énergétique, force est de constater que les effets positifs sont surestimés du fait de la non prise en compte des ER.

En outre, l'évaluation des effets des TNIC sur l'environnement souffre des faiblesses des analyses du cycle de vie (ACV) (Grisel et Osset, 2004), " méthodes d'évaluation environnementale qui permettent de quantifier les impacts d'un produit (qu'il s'agisse d'un bien, d'un service voire d'un procédé) sur l'ensemble de son cycle de vie ${ }^{19}$. La définition des " unités fonctionnelles » sur la base desquelles elles établissent leurs comparaisons sont discutables, comme le montre l'exemple dans l'encadré suivant. Elles ne donnent pas non plus de possibilité claire de débattre du poids relatif des impacts les uns par rapport aux autres - ici l'omission des déchets radioactifs, là les impacts sont noyés dans des " écopoints » dont l'arbitrage est opaque et technocratique (Bélem, 2005). Elles sont très coûteuses, une obligation d'y recourir équivaudrait donc à une élimination des petits acteurs, incapables de fournir les indications demandées. Les ACV peuvent alors fonctionner comme des barrières à l'entrée des marchés, renforçant les positions dominantes, et allongeant les détours de production qui sont justement à l'origine de la pollution grandissante... au contraire de l'objectif initial.

\section{Encadré : le problème de l'unité fonctionnelle, ou comment la méconnaissance de} l'analyse sociale contribue à produire des résultats aberrants.

Cherchant à établir le contenu en information de différents, Suh (2003) attribue 2 ko à une page écrite à la main (format A4), 563 ko au « Huckleberry Finn » de Mark Twain, 5 Mo aux CEuvres complètes de Shakespeare et ... 5 Mo pour 30 secondes de divertissement télévisuel.

Ce qui est comparé ici est "l'information ». Pour plus "d'objectivité " le chercheur à réduit l'information à sa mesure quantitative, ignorant l'aspect qualitatif. Mais une page originale de la Bible, par exemple, s'il en existait une, pourrait-elle être considérée comme contenant 2500 fois moins d'information qu'un show télévisuel que tout le monde oublié le lendemain ? Le concept " d'information » n'a manifestement pas le même sens quand on le mesure en " bits » et quand on le mesure en utilité sociale.

Cet exemple illustre bien la confusion qui règne. L'objectivité apparente de la mesure en « bits " est dangereusement trompeuse. Le biais est énorme et le risque de voir ce biais s'inscrire dans les politiques publiques est réel, puisque ce sont les ACV qui vont servir de point de comparaison.

Aujourd'hui l'usage des ACV n'est l'objet d'aucune investigation en sciences humaines. Personne n'essaie de les rendre transparentes et de les mettre au service de la démocratie. Le "découplage " tant évoqué prend aujourd'hui la forme d'un découplage non pas entre " croissance " et "planète » mais entre une classe aisée capable de se payer les technologies même lorsqu'elles seront devenues hors de prix et une classe pauvre qu'on aura privé de journaux pour des raisons écologiques.

A-t-on réfléchi pour mettre en place les instruments nécessaires à ce que le consommateur puisse faire un choix informé des conséquences sur l'environnement ? Quasiment pas, rien n'est fait en

\footnotetext{
18 Voir http://ec.europa.eu/information_society/activities/sustainable_growth/docs/com_2008_241_all_lang/com_2008_241_1_fr.pdf.
}

19 Source : http://www2.ademe.fr/servlet/KBaseShow?sort=-1\&cid=96\&m=3\&catid=13201. 
dehors du sigle indiquant la nécessité du tri (poubelle noire barrée) et l'indication de " l'écocontribution » correspondant à la prise en charge des déchets historiques, antérieurs à la mise en place de la directive DEEE. Comment est comprise l'écocontribution ? Personne ne s'en est soucié, pas plus de la lisibilité de la poubelle barrée, qui peut tout aussi bien signifier que si le tri est fait, alors le problème est réglé. Le problème est récurrent. Dans le domaine des emballages, le sigle circulaire vert avec deux flèches dans le même sens est souvent compris comme signifiant "recyclé » ou "recyclable » alors qu'il indique seulement que le fabricant de l'emballage contribue financièrement à l'éco-organisme Eco-Emballages.

\section{Conclusion}

Notre analyse de la situation, tant sur le plan micro que sur le plan macro, pose la question de savoir si les défis posés par l'ER au secteur des TNIC peuvent être dépassés. Avec la forte hausse du prix de l'énergie, les ER de type 1 et 2 vont être d'autant plus importants, puisque les économies monétaires dérivant de l'achat de produits et services moins gourmands en énergie vont être plus importantes - même si le ralentissement général de l'économie provoqué dans le même temps devrait endiguer la hausse du pouvoir d'achat, et donc limiter l'ER. Ensuite, les performances environnementales de ces produits et services ayant une grande marge de progression, on peut anticiper un fort effet de levier sur leur consommation, sans compter l'effet démultiplicateur d'Internet, fort vecteur d'accroissement de la consommation de biens et services. Pour Berkhout et Hertin (2004), la difficulté d'évaluer la contribution des TNIC au développement durable est due au fait qu'elles sont très imbriquées dans l'économie matérielle. D'après eux, à long terme l'impact environnemental net de la révolution de l'information dépendra de l'équilibre entre effets "dématérialisants" (par exemple les TNIC peuvent permettre la diffusion d'informations révélant les externalités de nos achats) et "rematérialisants » (les TNIC tirent et stimulent la croissance économique). Mais les entreprises n'ont aucun intérêt économique à diffuser les informations qui seraient mauvaises pour les ventes. Qui aura donc intérêt à mettre en lumière la dépendance croissante à des ressources épuisables ? II faut alors espérer que les acteurs seront de plus en plus sensibles aux questions environnementales et capables de modifier leurs comportements pour passer du statut de simples consommateurs à celui de citoyens responsables. La contribution positive des TNIC au développement durable sera au prix de ce pari, que peu de politiques ont jusqu'ici eu l'audace de relever.

La question qui n'a pas été ouverte, finalement, c'est la question de la régulation des besoins. Comment déterminer nos besoins autrement que par la publicité, qui vend depuis quelques années de véritables "styles de vie »? Quelles seraient les conséquences d'une augmentation massive du prix du pétrole et des matières premières sur les TNIC ? Quelles sont les priorités, poursuivre la fuite en avant technologique, garante de notre " compétitivité ", au prix de la planète et de la division croissante de notre société, ou commencer à réfléchir en termes plus larges ? La hausse continue du pouvoir d'achat conduit inévitablement à davantage de consommation. Ce que l'on nomme pudiquement « l'effet rebond » est finalement la conséquence directe des politiques de croissance mises en place depuis plus d'un siècle. Or cette politique a une contrepartie largement masquée - du moins tant que l'infrastructure n'est pas en place, que la dépendance n'est pas totale. Le consommateur, quand il est passé du téléphone fixe au mobile, a-t-il eu conscience qu'il contribuait à engager peu à peu toute la société sur un support de communication dépendant de ressources épuisables ? La discussion sur le "green IT » se focalise volontiers sur l'aspect énergétique, avec un horizon renouvelable. Mais dans le domaine de la matière ? Que se passerat-il si une matière clé vient à manquer ? Assisterons-nous, dans le domaine des TNIC, à ce que l'on a pu voir dans le domaine du transport courant 2008 et la flambée du baril de pétrole ? Avec quelles conséquences? 


\section{Bibliographie}

Aebischer, B., Roturier, J. (2006), Electricité et Internet : Le paradigme de l'iceberg, Bulletin de la Société Française de Physique, 154, 25-27, http://www.cepe.ethz.ch/publications/Aebischer_Roturier.pdf.

Attané, A. (2002), Pêcher, courir, trier ses déchets... pratiques de l'environnement ou rapport aux autres ? Ethnographie comparée des pratiques de l'environnement dans deux villes françaises (Cherbourg et Martigues), Europeae, 1-2, 261-282.

BAN et SVTC (2002), Exporting Harm - The high-tech trashing of Asia, http://www.ban.org/Ewaste/technotrashfinalcomp.pdf.

Barkera, T., Ekins, P., Foxon, T. (2007), The macro-economic rebound effect and the UK economy, Energy Policy, 35, 4935-4946.

Bélem, G. (2005), L'analyse de cycle de vie comme outil du développement durable, Les Cahiers de la Chaire de Responsabilité Sociale et de Développement Durable, ESG-UQAM.

Berkhout, F., Hertin, J. (2001), Impacts of Information and Communication Technologies on Environmental Sustainability: speculations and evidence - Report to the OECD.

Berkhout, F., Hertin, J. (2004), De-materialising and re-materialising: Digital technologies and the environment, Futures, 36, 903-920.

Berkhout, P.H.G., Muskens, J.C., Velthuijsen, J.W. (2000), Defining the rebound effect, Energy Policy, 28, 425-432.

Binswanger, M. (2001), Technological progress and sustainable development: What about the rebound effect?, Ecological Economics, 36, 119-132.

Birol, F., Keppler, J.H. (2000), Prices, technology development and the rebound effect, Energy Policy, 28, 457-469.

Cozzi, L. (2002), ICT and energy demand: An overview, IEA, Paris workshop, February, http://www.iea.org/textbase/work/workshopdetail.asp?WS ID=60.

Cremer, C. et al. (2003), Energy Consumption of ICT in Germany up to 2010, http://www.isi.fhg.de/e/eng/publikation/online/iuk/iuk-e.htm.

Daly, H., Cobb Jr, J. (1989), For the Common Good, Boston, Beacon Press.

Diamond, J., (2005), Collapse - How societies choose to fail or to succeed, New York, Penguin.

ECATT (2000), Benchmarking progress on new ways of working and new forms of business across Europe, Rapport final téléchargeable sur http://www.ecatt.com.

Faist Emmenegger, M. et al. (2003), LCA of the mobile communication system UMTS, SETAC, $11^{\text {th }}$ LCA Case Studies Symposium.

Flipo et al. (2006), Projet E-dechets - Ecologie des infrastructures numériques - Rapport final, Rapport de recherche pour l'Institut Télécom. http://etos.itsudparis.eu/rapports/INT_Flipo_Edechet_final_av06.pdf.

Gendron, C. (2002), Economie de l'environnement, économie écologique et sociologie économique de l'environnement : la nécessaire prise en compte des dimensions socialement construites du système économique et de la crise écologique, Europaea, 1-2, 187-212.

Greening, L.A., Greene, D.L., Difiglio, C. (2000), Energy efficiency and consumption the rebound effect: A survey, Energy Policy, 28, 389-401.

Grepperud, S., Rasmussen, I. (2004), A general equilibrium assessment of rebound effects, Energy Economics, 26, 261-282.

Grisel, L., Osset, P. (2004), L'Analyse du cycle de vie d'un produit ou d'un service - Applications et mise en pratique, AFNOR Editions.

Haan, P. de, Peters, A., Scholz, R.W., (2007), Reducing energy consumption in road transport through hybrid vehicles: investigation of rebound effects, and possible effects of tax rebates, Journal of Cleaner Production, 15, 1076-1084. 
Haas, R., Biermayr, P. (2000), The rebound effect for space heating: Empirical evidence from Austria, Energy Policy, 28, 403-410.

Herring, H. (1998), Does energy efficiency save energy? The economists debate, The Open University.

Herring, H. (2004), The rebound effect and energy conservation, in Cleveland, C. (Ed.), The Encyclopedia of Energy, Academic Press/Elsevier Science.

Herring, H., Roy, R. (2002), Sustainable services, electronic education and the rebound effect, Environmental Impact Assessment Review, 22, 525-542.

Herring, H., Roy, R. (2007), Technological Innovation, energy efficient design and the rebound effect, Technovation, 27, 194-203.

Hilty, L.M. (2008), Information technology and sustainability: Essays on the relationships between information technology and sustainable development, Norderstedt, Books on Demand.

IEA (2005), The experience with energy efficiency policies and programmes in IEA countries, Paris, IEA.

Kuehr, R., Williams, E. (2003), Computers and the environment - Understanding and managing their impacts, Kluwer Academic Publishers, United nations University.

Laitner, J.A. "Skip" (2003), Information technology and U.S. energy consumption, Journal of Industrial Ecology, 6(2), 13-24.

Mizobuchi, K. (2008), An empirical study on the rebound effect considering capital costs, Energy Economics, 30(5), 2486-2516.

Ostroff, J. (2007), Les déchets électroniques : une menace mondiale, Hiver 2004-2005, http://www.cec.org/trio/stories/index.cfm?ed=14\&ID=157\&varlan=francais, Trio.

Plepys, A. (2002), The grey side of ICT, Environmental Impact Assessment Review, 22, 509-523.

PNUE (2005), Les déchets électroniques : La face cachée de l'ascension des technologies de l'information et des communications, Bulletin d'Alerte Environnementale, http://www.grid.unep.ch/product/publication/download/ew_ewaste.fr.pdf.

Schipper, L., Grubb, M. (2000), On the rebound? Feedback between energy intensities and energy uses in IEA countries, Energy Policy, 28, 367-388.

Small, K.A., Van Dender, K. (2007), Fuel efficiency and motor vehicle travel: The declining rebound effect, The Energy Journal, 28(1), 25-51.

Sorrell, S., Dimitropoulos, J. (2005), An assessment of evidence for a 'rebound effect' from improvements in energy efficiency, Scoping Note, SPRU, University of Sussex.

Suh, S. (2003), Materials and energy basis of information - The weight of information, SETAC, $11^{\text {th }}$ LCA Case Studies Symposium.

Toxic Link (2003), Scrapping the high-tech myth - computer waste in India, http://www.toxicslink.org/pub-view.php?pubnum=37.

US EPA (2001), Electronics : A new opportunity for waste prevention, reuse, and recycling, rapport téléchargeable sur http://www.ecyclingtools.com/documents/EPAbkgd.pdf.

Wuppertal Institut (2003), Digital Europe, rapport téléchargeable sur www.digital-eu.org.

Zuindeau, B. (2005), La « loi de Kuznets »: De l'économie de la répartition à l'économie de l'environnement, Communication au Colloque International de l'Association Charles Gide pour l'Étude de la Pensée Économique, 22-24 septembre, Lille. 